\title{
Acoustic detection of microbubble formation induced by enhanced optical breakdown of silver/dendrimer nanocomposites
}

\author{
Susanne M. Milas, Jing Yong Ye, ${ }^{a), b)}$ Theodore B. Norris, ${ }^{a)}$ Lajos P. Balogh, ${ }^{c}$ \\ James R. Baker, Jr., ${ }^{\text {d) }}$ Kyle W. Hollman, Stanislav Emelianov, and Matthew O'Donnell \\ Department of Biomedical Engineering, University of Michigan, 3304 G, G. Brown Labs, Ann Arbor, \\ Michigan 48109-2125
}

(Received 24 June 2002; accepted 18 December 2002)

\begin{abstract}
We utilize a real-time acoustic technique, based on pulse-echo measurements to detect formation of microbubbles in an aqueous solution of a silver/dendrimer nanocomposite (DNC). Wave-field plots of successive recordings illustrate the generation and behavior of bubbles created by the optical breakdown process. A significant threshold reduction is achieved with DNC particles compared to its host dendrimer, enabling a diverse field of low-threshold breakdown applications. (C) 2003 American Institute of Physics. [DOI: 10.1063/1.1544657]
\end{abstract}

Laser-induced optical breakdown (LIOB) with femtosecond pulsed lasers is utilized in diverse applications, including biomedical systems, material characterization, and data storage. ${ }^{1-4}$ LIOB parameters and behavior have been investigated extensively. ${ }^{5-9}$ It occurs when sufficiently high threshold fluence is attained at the laser focus, inducing plasma formation. Plasma formation leads to nonlinear energy absorption and measurable secondary effects that include shock-wave emission, heat transfer, and bubble formation (i.e., photodisruption). The presence and magnitude of these breakdown attributes are used to determine a material's LIOB threshold.

Dendrimers are highly branched spherical macromolecules that provide templates for guest molecules ${ }^{10}$ to form dendrimer nanocomposite (DNC) particles. By trapping metallic domains in dendrimers, the LIOB threshold of the organic host in water and biological tissue can be decreased. Enhanced electric fields are established around nanocomposite particles with these metallic guests that significantly reduce breakdown fluences. Previous investigations ${ }^{11}$ have measured the breakdown fluence of aqueous DNC solutions as low as $9.5 \mathrm{~mJ} / \mathrm{cm}^{2}$. Optical limiting and third-harmonicgeneration (THG) techniques can explore some nonlinear optical properties of these particles and their aggregates; ${ }^{11,12}$ yet they provide only limited information about the photodisruption.

We report here a real-time acoustic technique for direct detection of LIOB thresholds in various liquids, ${ }^{13}$ and its application to the study of DNC-enhanced breakdown. Femtosecond laser pulses, produced by a $250-\mathrm{kHz}$ regeneratively amplified Ti:sapphire laser $(\lambda=793 \mathrm{~nm})$, are directed through the bottom of a small cylindrical tank with an inner diameter of $14 \mathrm{~mm}$ and height of $10 \mathrm{~mm}$. The laser focus (10

\footnotetext{
${ }^{a)}$ Center for Ultrafast Optical Science, University of Michigan, 2200 Bonisteel Boulevard, Ann Arbor, Michigan 48109-2099.

${ }^{b}$ Electronic mail: jyye@eecs.umich.edu

${ }^{c}$ Center for Biologic Nanotechnology, the Department of Internal Medicine, and Macromolecular Science \& Engineering Program, University of Michigan, Ann Arbor, Michigan 48109-0533.

${ }^{d)}$ Center for Biologic Nanotechnology and the Department of Internal Medicine, University of Michigan, Ann Arbor, Michigan 48109-0533.
}

$\mu \mathrm{m}$ full width at half maximum) is positioned using THG measurements at the tank's bottom inside surface. When sufficiently intense laser pulses are applied to this interface, nonlinear absorption ablates a localized volume, that is, a photodisruption occurs. In our technique, a tightly focused, single-element, ultrasonic transducer (center frequency 50 $\mathrm{MHz}, 4.1-\mathrm{mm}$ focal depth, 3-mm diameter) is positioned such that its focus coincides axially and laterally with this laser focus. This custom-built polymer transducer is one focal length axially from the tank bottom.

During photodisruption, a bubble forms, resulting in a high-velocity shock wave that propagates spherically outward from the effective point source. After traveling a few wavelengths from the source, this wave can be considered a broadband pressure wave propagating toward the transducer, that is, an acoustic emission. To characterize the bubble, we actively probe the tank's bottom surface via pulse-echo measurements from the same transducer. If a bubble forms, the signal will have a pulse (i.e., reflection) from the top surface of the bubble and a pulse from the tank bottom (if either acoustic attenuation by the bubble is small or the acoustic focal spot is larger than the bubble size). The time difference between these pulses is quantified as an acoustic shift on the order of nanoseconds. By viewing acoustic shifts in consecutive pulse-echoes (i.e., wave-field plots), bubble formation and subsequent behavior can be visualized.

Properties of two aqueous solutions containing practically identical dendrimer concentrations were evaluated. A carboxyl terminated, ethylenediamine core, generation five poly(amidoamine) (PAMAM) dendrimer served as a template for the silver nanocomposite. ${ }^{14}$ $\left\{\operatorname{Ag}(0)_{97}\right.$-PAMAM_E5.5COOH $\}$, a silver-dendrimer hybrid DNC, $\left[\{\operatorname{Ag}(0)\}_{\mathrm{E}}\right.$ : for short $]$, composed of $25.66 \mathrm{mg} / \mathrm{g}$ dendrimer plus $6.116 \mathrm{mg} / \mathrm{g}$ immobilized $\operatorname{Ag}(0)_{\mathrm{E}}$ was used as a nanocomposite. To provide the same optical density of the chromophores in the control, an aqueous solution of the template dendrimer was used $(c=23.77 \mathrm{mg} / \mathrm{g})$. After optical alignment, the acoustical tank was filled with the test liquid, while laser pulses were blocked with a mechanical shutter. Each data record consisted of opening the shutter and recording approximately one second of pulse-echo signals (2700 

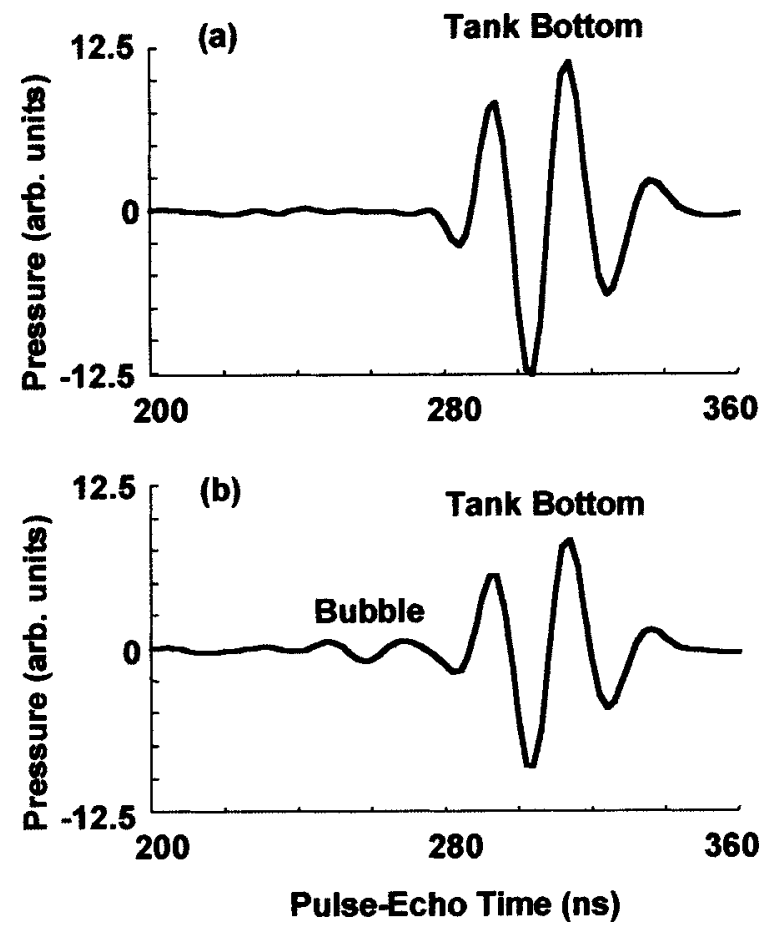

FIG. 1. Two pulse-echos from pure dendrimer solution irradiated with $50-\mathrm{mW}$ laser pulses $\left(260 \mathrm{~mJ} / \mathrm{cm}^{2}\right.$ per pulse). Pulse-echo (a) is a reflection from only the tank bottom. Pulse-echo (b) shows the formation of a bubble. The acoustic shift is measured as the time between the second-zero crossings of the two pulses in (b). For this pulse-echo, a 34-ns shift is observed, corresponding to a $25.5-\mu \mathrm{m}$ bubble.

consecutive recordings). Each recording was $360 \mathrm{~ns}$ long, triggered by the transducer's pulser with a repetition rate of $2.44 \mathrm{kHz}$. Acoustic emissions can occur on each laser firing (250-kHz repetition rate); however, the recording of these signals was not synchronized with the pulse-echo data reported here.

Wave-field plots of consecutive pulse-echo recordings enable us to observe bubble formation and behavior directly. While the mechanical shutter blocks the laser pulses, the pulse-echo signal simply represents an acoustic reflection from the tank bottom. This signal persists when the laser is unblocked if its power lies below the liquid's LIOB threshold. Once threshold is attained, significant changes are observed. The presence of additional acoustic reflections is used for our threshold estimates.

For a pure dendrimer aqueous solution, the optical threshold (average laser power) at which significant acoustic power is reflected from a microbubble was found to be 50 $\mathrm{mW}\left(260 \mathrm{~mJ} / \mathrm{cm}^{2}\right.$ per pulse). Figure 1 shows two pulse-echo recordings taken at the dendrimer's threshold power level; the first illustrates laser blockage and the second is during bubble growth. By comparing these lines, it is clear that a bubble has formed with a 34-ns acoustic shift, corresponding to a $25.5-\mu \mathrm{m}$ bubble. Figure 2(a) is a wave-field plot illustrating this bubble's formation and adherence to the tank surface. Initially, only the reflection from the tank bottom is observed, corresponding to laser blockage. When the bubble forms, a new acoustic pulse emerges above this reflection and gradually shifts toward the transducer. As time progresses, the shift increases, confirming bubble growth. The final extent of this shift provides a direct measure of axial bubble size.
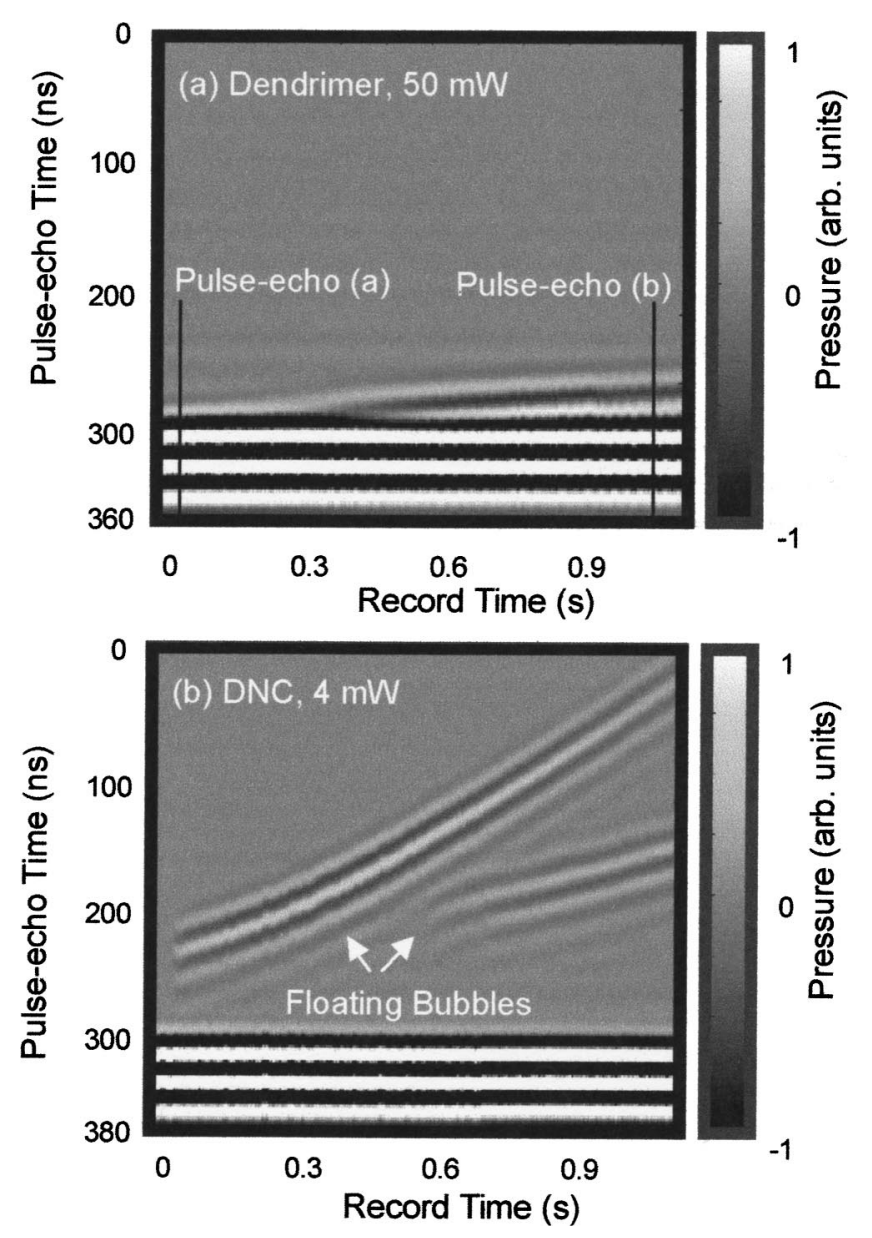

FIG. 2. (a) Wave-field plot of dendrimer solution irradiated with 50-mW laser pulses $\left(260 \mathrm{~mJ} / \mathrm{cm}^{2}\right.$ per pulse). When the laser is unblocked (time origin approximates shutter opening), a bubble forms, adheres to the tank bottom, and grows. The location of the two pulse-echos from Fig. 1 are noted. (b) Wave-field plot of $\{\operatorname{Ag}(0)\}_{\mathrm{E}}$ solution irradiated with 4-mW laser pulses $\left(21 \mathrm{~mJ} / \mathrm{cm}^{2}\right.$ per pulse). When the laser is unblocked, a bubble forms and floats upward toward the transducer. Around $0.6 \mathrm{~s}$, a second bubble forms and also travels upward. Each liquid has slightly different acoustical properties, resulting in different sound propagation speeds within the liquid. To accommodate this, longer data records (around $20 \mathrm{~ns}$ ) were acquired for measurements in $\{\operatorname{Ag}(0)\}_{E}$ solutions than in pure dendrimer.

The aqueous solution of $\{\operatorname{Ag}(0)\}_{\mathrm{E}}$ was characterized using the same procedure. The LIOB threshold determined acoustically for this $\{\mathrm{Ag}(0)\}_{\mathrm{E}}$ is $4 \mathrm{~mW}\left(21 \mathrm{~mJ} / \mathrm{cm}^{2}\right.$ per pulse), over an order of magnitude lower than the threshold of the pure dendrimer solution. We note that in previous studies for pure water, a threshold of $250 \mathrm{~mW}\left(1.3 \mathrm{~J} / \mathrm{cm}^{2}\right.$ per pulse) was obtained, more than 50 times higher than the threshold for the DNC solution. ${ }^{13}$ This result provides an unquestionable advantage of using DNC particles with a low threshold for photodisruption. Figure 2(b) illustrates the acoustic events when the DNC solution is irradiated with laser pulses close to its LIOB threshold. Initially, no bubble is present. Once the shutter opens, two distinct events occur. The bubbles do not adhere to the tank surface as seen for pure dendrimer; instead, they float upward toward the transducer. As bubbles move toward the transducer, they exit the transducer's $250 \mu \mathrm{m}$ depth of field and pulse-echo measurements decrease in intensity.

A range of laser powers was investigated for each aqueous solution. For each laser power, the average reflected 


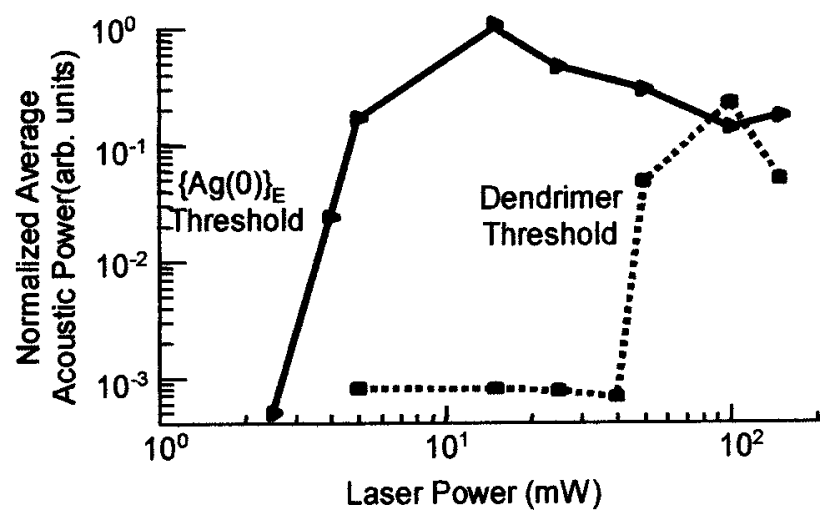

FIG. 3. Normalized average acoustic power vs laser power. LIOB threshold values are marked for $\{\operatorname{Ag}(0)\}_{\mathrm{E}} \mathrm{DNC}$ and PAMAM dendrimer; $4 \mathrm{~mW}(21$ $\mathrm{mJ} / \mathrm{cm}^{2}$ per pulse) and $50 \mathrm{~mW}\left(260 \mathrm{~mJ} / \mathrm{cm}^{2}\right.$ per pulse), respectively.

acoustic power from the top of the bubble was calculated as the sum of the squared amplitude, averaged over time. This provides a quantifiable measure of the average acoustic power associated with nonbreakdown and breakdown events. Figure 3 shows the dramatic increase in average acoustic power for data records containing optical breakdowns. At high laser powers, acoustic shadowing due to bubble generation accounts for the slight decline in acoustic power. Figure 3 , furthermore, illustrates the ability to manipulate LIOB threshold values based on the solution's molecular attributes. This unlocks enormous potential in many applications. For instance, in biomedical systems, DNC particles can be biochemically targeted to sites where localized photodisruption can be induced, either releasing encapsulated therapeutics or ablating aberrant cells (such as cancer cells or tissue). Furthermore, bubbles resulting from photodisruption can be manipulated with either acoustic or optical sources for enhanced therapeutic effects, ${ }^{3}$ and probed with high frequency ultrasound to validate therapeutic efficacy.
In conclusion, we have presented an acoustic technique to detect LIOB thresholds in solutions. Furthermore, we have used this technique to quantify the significant reduction in LIOB threshold when DNC particles were introduced to the environment.

This project is supported in part by the National Institutes of Health under NOI-CO-97111, HL-47401, DK47324, HL-67647, by the U.S. Department of Energy under FG01-00NE22943, and by the Whitaker Foundation.

${ }^{1}$ T. Juhasz, F. H. Loesel, R. M. Kurtz, C. Horvath, J. F. Bille, and G. Mourou, IEEE J. Quantum Electron. 5, 902 (1999).

${ }^{2}$ A. Vogel, Appl. Phys. B: Lasers Opt. 68, 271 (1999).

${ }^{3}$ G. J. R. Spooner, A. R. Williams, D. L. Miller, and G. Marre, Proc. SPIE 3914, 207 (2000).

${ }^{4}$ C. B. Schaffer, N. Nishimura, and E. Mazur, Proc. SPIE 3451, 2 (1998).

${ }^{5}$ A. Vogel, J. Noack, K. Nahen, D. Theisen, S. Busch, U. Parlitz, D. X. Hammer, G. D. Noojin, B. A. Rockwell, and R. Birngruber, Appl. Phys. B: Lasers Opt. 68, 271 (1999).

${ }^{6}$ Q. Feng, J. V. Moloney, A. C. Newell, E. M. Wright, K. Cook, P. K. Kennedy, D. X. Hammer, B. A. Rocwell, and C. R. Thompson, IEEE J. Quantum Electron. 33, 127 (1997).

${ }^{7}$ J. Noack, D. X. Hammer, G. D. Noojin, B. A. Rockwell, and A. Vogel, J. Appl. Phys. 83, 7488 (1998).

${ }^{8}$ D. X. Hammer, R. J. Thomas, M. Frenz, E. D. Jansen, G. D. Noojin, S. J. Diggs, J. Noack, A. Vogel, and B. A. Rockwell, Proc. SPIE 2681, 437 (1996)

${ }^{9}$ E. N. Glezer, C. B. Schaffer, N. Nishimura, and E. Mazur, Opt. Lett. 22, 1817 (1997)

${ }^{10}$ L. Balogh, R. Valluzzi, G. L. Hagnauer, K. S. Laverdure, S. P. Gido, and D. A. Tomalia, Nanoparticle Res. 1, 353 (1999).

${ }^{11}$ J. Y. Ye, L. Balogh, and T. B. Norris, Appl. Phys. Lett. 80, 1713 (2002).

${ }^{12}$ R. Ispasoiu, L. Balogh, O. P. Varnavski, D. A. Tomalia, and T. Goodson III, J. Am. Chem. Soc. 122, 11005 (2000).

${ }^{13}$ S. M. Milas, J. Y. Ye, T. B. Norris, K. W. Hollman, S. Emelianov, and M. O'Donnell, IEEE Trans. Ultrason. Ferroelectr. Freq. Control (in press).

${ }^{14}$ L. Balogh, D. R. Swanson, D. A. Tomalia, G. L. Hagnauer, and A. T. McManus, Nano Lett. 1, 18 (2001) 